\title{
77-year old woman with free-floating thrombus in abdominal aorta, causing acute renal dysfunction
}

\author{
Diana Kupczynska, Piotr Barc, Maciej Antkiewicz, Piotr Szyber \\ Department and Clinic of Vascular, General and Transplantation Surgery, Jan Mikulicz-Radecki Medical University Hospital, \\ Wroclaw, Poland
}

\begin{abstract}
The aim of this paper is to present extremely rare case of 77-year old woman with free floating thrombus (FFT) in abdominal aorta obliterating superior mesenteric and right renal artery. The patient developed acute renal dysfunction. FFT was entirely removed surgically from aorta, renal arteries and celiac arteries obtaining good outflows. Appropriate treatment based on in-depth patient's condition evaluation prevented severe, potentially fatal complications.
\end{abstract}

Key words: renal artery, superior mesenteric artery, abdominal aorta, free-floating thrombus

Acta Angiol 2017; 23, 3: 135-138

\section{Introduction}

During on-call time in the angiosurgical ward we often deal with embolisms that are a consequence of hypercoagulation. The causes of the state are most frequently: atrial fibrillation, coagulopathies, injuries, cancers or instrumentation of vessels [I]. A blood clot usually forms in the aorta in the atherosclerotic internal elastic lamina [2]. It is rare to observe a blood clot in the lumen of an pathologically unchanged vessel. Then only a part of the thrombus is affixed to the aorta or trapped in it whilst the rest of it floats freely in its lumen under the influence of blood flowing through the artery. $\mathrm{Kim}$ and others used a term free-floating thrombus (FFT) to describe the state [3]. So far around 100 of such cases were described and most of the cases concerned the thoracic aorta whilst FFT of the abdominal aorta hardly ever presents. The clinical picture differs depending on the morphology and localization of the thrombus. The course of disease may be asymptomatic and lead to the serious cerebrovascular, visceral and peripheral embolism. These complications occur far more commonly than in non-floating thrombi. In the case of abdominal aorta a thrombus is usually located in the supra-renal area and causes renal infarction, splenic infarction and superior mesenteric artery embolism. Less frequently a thrombus is located in sub-renal aorta which can cause an acute lower limb ischaemia [2, 3]. The above described states can lead to very serious complications such as necrosis of the intestines, limbs or even death [4].

We have described the case of the floating thrombus in the aorta causing a simultaneous closure of left renal artery and superior mesenteric artery. In the course of the ischaemia the acute renal failure overlapping chronic renal disease and slowly developing intestinal ischaemia (because of only partial closure of the lumen of superior mesenteric artery) was developed. According to our knowledge it is one of the rarest clinical situations among previously-described FFTs which is extremely peculiar itself and, at the same time, carries a high-level risk of death. The patient signed a consent form about publication of her data in this article.

\section{Case study}

77-year old woman with atrial fibrillation and chronic renal failure treated in the local hospital for abdominal 

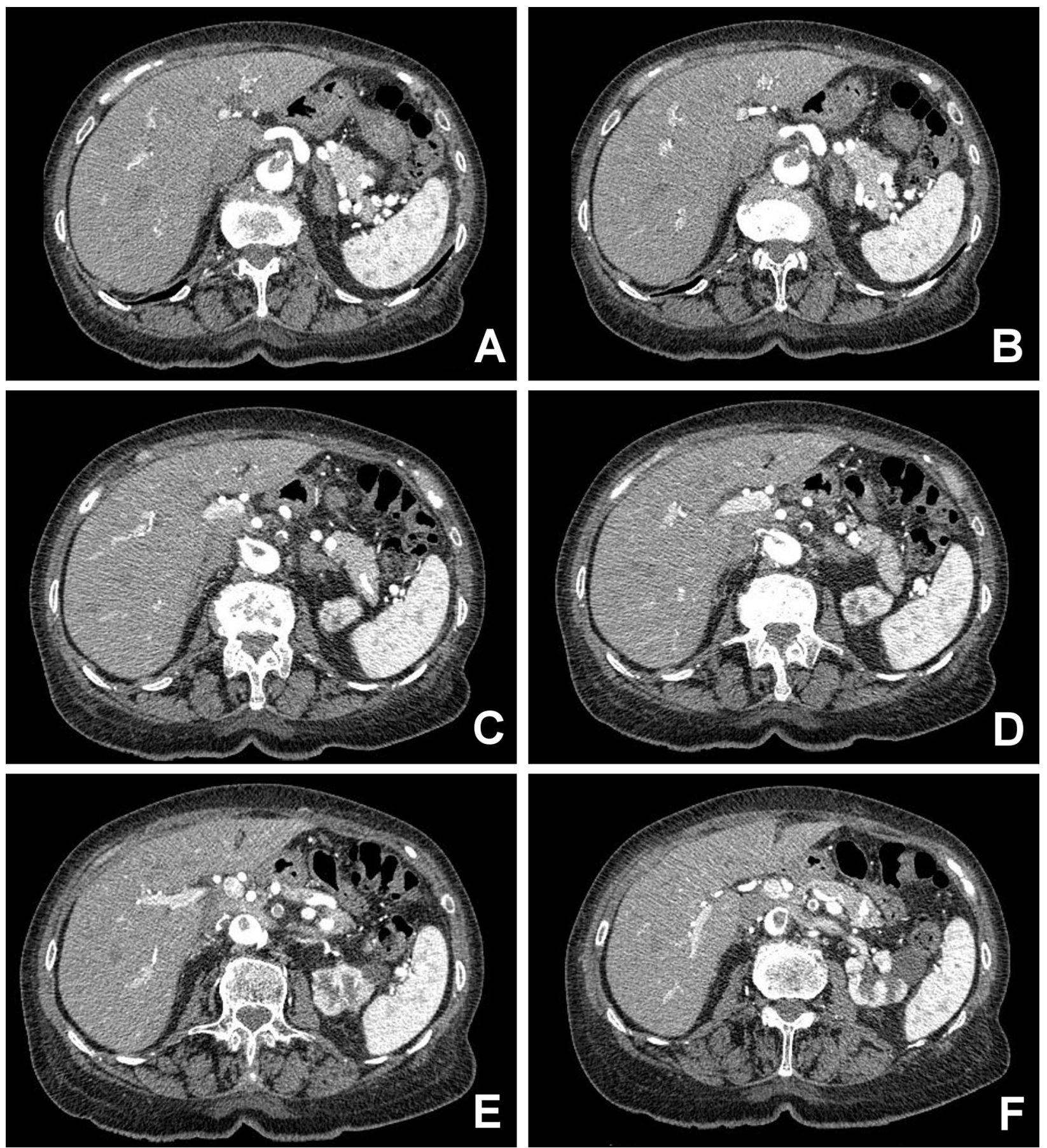

Figure I. Computed tomography scans show free-floating thrombus in abdominal aorta. A, B. FFT obliterates superior mesenteric artery; C, D. FFT obliterates right renal artery; E, F. FFT in sub-renal aorta

pain. During hospitalization remarkably elevated creatinine level was observed. For that reason, as well as the increasing abdominal symptoms, the patient underwent ultrasound examination on the basis of which the floating thrombus in the aorta was suspected. With this diagnosis the patient went to our hospital. The creatinine level at admission was $3.68 \mathrm{mg} / \mathrm{dL}$. The repeated ultrasound examination with Duplex-Doppler option confirmed abnormal blood flow in the lumen of the aorta in the area of renal arteries. Due to a low objectivity of the examination, CT angiography of abdominal aorta was performed (Fig. IA-F). The examination revealed a $5 \mathrm{~cm}$ long thrombus extending from superior mesenteric artery, penetrating and obliterating superior mesenteric artery (Fig. IA, B) and penetrating and obliterating right renal artery on a length of $1.5 \mathrm{~cm}$ (Fig. IC, D). 
On the basis of the above-mentioned examination and because of deteriorating laboratory features of impairment of renal function and possibility of developing intestinal ischaemia the decision about immediate surgical treatment was made. The operation was performed under general anaesthesia. After opening the abdominal cavity no intestinal necrosis was found and intestinal peristalsis was preserved. After cutting through back peritoneum layer and lifting the pancreas and the duodenum the further part of the aorta was dissected. After placing the ribbons on and administering 5000 units of heparin overall, the clamps were placed on aorta above the celiac trunk and just above aortic bifurcation. The aorta was opened by making the longitudinal cut above the thrombus. It was entirely removed from aorta, renal arteries and celiac arteries obtaining good outflows. Satynsky clamp was placed tangentially on aorta which was stitched with a Dacron patch. After the surgery the patient was administered 20000 units/24 h heparin intravenously under the control of APTT. During the subsequent events of atrial fibrillation with ventricular action of 100-150/min were observed. On day 5 of hospitalization the patient was transferred to the department of cardiology, where transesophageal echocardiography and successful cardioversion was performed. 6, 12, 18 months follow-up with abdominal ultrasonography and basic laboratory tests was implemented. Each time ultrasonography examination showed no abnormalities within aortic region. Kidneys, liver and intestines functions were normal. Creatinine level was: $1.17 \mathrm{mg} / \mathrm{dL}, 1.34 \mathrm{mg} / \mathrm{dL}$, $1.04 \mathrm{mg} / \mathrm{dL}$ respectively.

\section{Discussion}

In order to assess a thrombus in aorta the most optimal examination would be angio-CT usually preceded after the ultrasound examination [I, 3]. In the case of FFT placed in the thoracic aorta the alternatives are MR, angio-MR and transesophageal echocardiography in the case of FFT placed in the thoracic aorta [2]. Because of a high risk of embolism FFT is a life-threatening state. Thus it requires implementation of a proper therapy. Unfortunately, by virtue of its rarity it is hard to determine an optimal behaviour. Kim and others suggest starting the therapy with systemic anticoagulation with low-molecular-weight heparin. Only in case of ineffectiveness of the treatment or thromboembolic complications it is necessary to implement surgical procedures. Out of their seven patients treated with heparin in as many as three cases it came to the complete displacement of the thrombus which demanded immediate surgery [3]. Sanson and others are of the opposite view. According to them systemic anticoagu- lation increases the risk of peripheral embolism when the floating thrombus begins to disintegrate. Thereby in case of FFT they suggest the implementation of an early surgical treatment [2]. In cases of a massive thrombus extending from aortic arch to subrenal aorta the thoracotomy and revision of aorta followed by embolectomy may be necessary.

By virtue of a high risk of peripheral embolism additional thrombectomy of iliac, femoral and popliteal arteries are worth considering [5]. In case of FFT, endovascular methods of treatment are also considered. Stent graft implantation in the area of thrombus causes its immobilisation by the vessel wall and prevents relapse due to atherosclerosis and other pathologies in that region. Disadvantage of the procedure is high risk of mobilisation of a thrombus, and peripheral embolism as a result. Possibility of such an incident can be decreased by closing potential ways of migrating thrombus such as common femoral artery and one of common iliac arteries with Fogarty catheter. After such a protection embolectomy of aorta with Fogarty catheter can be performed from femoral access (femoral superficial artery) on the opposite site and implantation of stent graft can be performed [6]. Unfortunately this procedure cannot be used in case of celiac arteries because of exceptionally high risk of their closure.

By virtue of possibility of distant complications and recurrence of FFT those patients demand long-term observation (including angio-CT, usually after six months and anticoagulation treatment).

It is usually achieved with the help of vitamin K antimetabolites under control of INR on the level $2[3,5]$. Because of very few cases of FFT in the world the exact etiology of the occurrence remains unclear. Past experiences show that it is linked to patient's predisposition to hypercoagulation. Kim and others demonstrated that in each of the seven cases of FFT, during thrombectomy, thrombus came from lumbar arteries.

By virtue of the lack of other risk factors they legitimised turbulent blood flow as the reason of their formation [3]. Kamiya and others considered as a reason increased activity of platelets as a consequence of patient's thromboangiitis obliterans [7]. In turn, Akagi and others in their work mentioned the case of the thrombus being most likely complication after chemotherapy of stomach cancer. Symptoms (abdominal pain and intermittent claudication of a calf) appeared on the third day of cisplatin ingestion [6]. Floating thrombus was also described in the case of 82 year old patient with iatrogenic Cushing's disease (after treatment for RA). The pathomechanism leading to hypercoagulation in this case was probably the increased blood platelet and von Willebrand factor activity caused by glycocortycosteroids [I]. 
The risk of FFT is also higher for patients with coagulation system abnormalities such as excess of factor VIII [4], excess of antithrombin III, protein S, protein C [2].

\section{Conclusions}

On the basis of the above-described case study it can be easily noticed that a floating thrombus in aorta can lead in a very short time to serious complications and it can also be the direct cause of health loss or even death. Any suspected case of FFT requires immediate diagnosis in vascular surgery department and implementation correct and appropriate treatment based on in-depth patient's condition evaluation, imaging findings and therapeutical possibilities.

\section{References}

I. Kim DH, Choi DH, Lee YM, et al. Massive thoracoabdominal aortic thrombosis in a patient with iatrogenic Cushing syndrome. Korean J Radiol. 2014; 15(5): 637-640, doi: 10.3348/ /kjr.20 I4.15.5.637, indexed in Pubmed: 25246825.
2. Sanon S, Phung MK, Lentz R, et al. Floating, non-occlusive, mobile aortic thrombus and splenic infarction associated with protein C deficiency. J Am Soc Echocardiogr. 2009; 22(12): 1419.el-1419. 3, doi: 10.1016/j.echo.2009.06.004, indexed in Pubmed: 19647398

3. Kim S, Hwang J, Lee J, et al. Free floating thrombus of the aorta: an unusual cause of peripheral embolization. J Korean Surg Soc. 20I I; 80(3): 204-2II, doi: 10.4I74/jkss.20II.80.3.204.

4. Bosma J, Rijbroek A, Rauwerda JA. A rare case of thromboembolism in a $2 \mathrm{I}$-year old female with elevated factor VIII. Eur J Vasc Endovasc Surg. 2007; 34(5): 592-594, doi: 10.1016/j. ejvs.2007.05.019, indexed in Pubmed: 1766967I.

5. Takagi $\mathrm{H}$, Mori $\mathrm{Y}, \mathrm{Umeda} \mathrm{Y}$, et al. Surgical treatment of thoracoabdominal aortic mural and floating thrombi extending to infrarenal aorta. J Vasc Surg. 2003; 37(6): |324-1327, indexed in Pubmed: 12764284.

6. Akagi $\mathrm{H}$, Irie $\mathrm{H}, \mathrm{Nakao} \mathrm{Y}$, et al. Endovascular treatment of acute abdominal aortic thrombosis after cisplatin-based chemotherapy. J Vasc Med Surg. 20 I 3; I (2): I I I, doi: 10.4 I72/2329-6925. 1000 I I I.

7. Kamiya C, Deguchi J, Kitaoka T, et al. Obstruction of the superior mesenteric artery due to emboli from the thoracic aorta in a patient with thromboangiitis obliterans. Ann Vasc Dis. 2014; 7(3): 320-324, doi: 10.3400/avd.cr.14-00035, indexed in Pubmed: 25298837. 